\title{
Lifesaving Emergency Sternotomy in Traumatic Cardiac Tamponade in a Teaching Hospital in Ghana: Survival of Two Rare Cases
}

\author{
Isaac Okyere ${ }^{1,2^{*}}$, Samuel Gyasi Brenu ${ }^{3}$, Perditer Okyere ${ }^{2,4}$ \\ ${ }^{1}$ Cardiovascular and Thoracic Surgery Unit, Department of Surgery, School of Medicine and Dentistry, College of Health Sciences, \\ Kwame Nkrumah University of Science and Technology, Kumasi, Ghana \\ ${ }^{2}$ Komfo Anokye Teaching Hospital, Kumasi, Ghana \\ ${ }^{3}$ Departemnt of Surgery, School of Medical Sciences, University of Cape Coast, Cape Coast, Ghana \\ ${ }^{4}$ Department of Medicine, School of Medicine and Dentistry, College of Health Sciences, Kwame Nkrumah University of Science \\ and Technology, Kumasi, Ghana \\ Email: ${ }^{\star}$ rokyere@yahoo.com
}

How to cite this paper: Okyere, I., Brenu, S.G. and Okyere, P. (2020) Lifesaving Emergency Sternotomy in Traumatic Cardiac Tamponade in a Teaching Hospital in Ghana: Survival of Two Rare Cases. World Journal of Cardiovascular Surgery, 10, 33-40. https://doi.org/10.4236/wjcs.2020.103005

Received: December 25, 2019

Accepted: March 24, 2020

Published: March 27, 2020

Copyright $\odot 2020$ by author(s) and Scientific Research Publishing Inc. This work is licensed under the Creative Commons Attribution International License (CC BY 4.0).

http://creativecommons.org/licenses/by/4.0/

\begin{abstract}
Introduction: Patients presenting with cardiac injuries from gunshot wounds and blunt chest trauma have high mortality, without any observed survival benefit when presenting with cardiac tamponade. Cardiac tamponade is a life-threatening hemodynamically significant compression of the heart by a sudden or gradual accumulation of collections in the pericardial space that incites and overrides the body's compensatory mechanism. Clinical Case: We present and discuss the successful management and survival of two patients with traumatic cardiac tamponade from gunshot wounds to the precordium who underwent successful lifesaving median sternotomy at a Teaching Hospital in Ghana with a new Cardiovascular and Thoracic Surgery Unit. Discussion: Usually the diagnosis of cardiac tamponade from traumatic haemopericardium is made by clinical findings which though may not always be present especially after blunt chest trauma. EFAST is a reliable tool for diagnosing and following cardiac tamponade. Median sternotomy is the standard procedure in these patients to access and repair cardiac injury either with or without cardiopulmonary bypass. Conclusion: Emergency median sternotomy in patients with cardiac tamponade from chest trauma especially after EFAST diagnosis can be lifesaving even in less resourced centres.
\end{abstract}

\section{Keywords}

Median Sternotomy, Cardiac Tamponade, Chest Trauma, Emergency 


\section{Introduction}

Prior to 1882 when the first ever successful myocardial suturing was done, traumatic cardiac injuries were seen as death sentences [1] with injuries ranging from contusions to chamber wall ruptures as well as great vessel and valvular injuries [2]. These cardiac injuries may lead to death via exsanguination or development of cardiac tamponade [1]. Cardiac tamponade is a life-threatening hemodynamically significant compression of the heart by a sudden or gradual accumulation of collections in the pericardial space that incites and overrides the body's compensatory mechanism. It is more life-threatening when it follows chest trauma with increased mortality.

\section{Case 1}

A 32-year-old male patient was rushed to the accident and emergency centre of the Komfo Anokye Teaching Hospital in Ghana after he was allegedly shot in the chest. He reported with complaints of severe chest pain but with no associated dyspnoea, orthopnoea, palpitations or cough. On arrival, he was hemodynamically stable with of BP 120/80 mmHg, PR $126 \mathrm{bpm}, \mathrm{RR} 26 \mathrm{cpm}$ and GCS 15/15. Chest was clinically clear but had multiple gunshot pellet wounds over his precordium. All other organ systems were clinically normal. E-FAST showed a moderate pericardial effusion with collapse of the right ventricle (Tamponade) but negative haemoperitoneum. The sonographer could not visualize the left hemidiaphragm.

An initial impression of moderate hemopericardium $2^{\circ}$ precordial gunshot injury was made. Electrocardiogram done showed normal QRS complex height while a chest $\mathrm{x}$-ray showed multiple pellets in the chest and abdomen with cardiomegaly and obliterated left costophrenic angle indicating the presence of haemothorax. Laboratory investigations done showed $\mathrm{Hb}$ of $15.5 \mathrm{~g} / \mathrm{dl}$, WBC$14.07 \times 10^{3} / \mathrm{uL}$, Platelets $-246 \times 10^{3} / \mathrm{uL}$ and normal renal and liver function tests. After 4 hours of resuscitation, his BP had dropped to 100/60 $\mathrm{mmHg}$ and the heart rate to 119 beats per minute. He was therefore consented for exploratory Median sternotomy because of the suspecting tamponading haemopericardium.

He was immediately rushed to the operating theatre. After general anaesthesia with endotracheal intubation in supine position, the chest was entered via a median sternotomy. The findings included:

- Multiple precordial and subcutaneous pellets

- Tense anterior pericardium

- Intact intra-cardiac organs and vessels

A midline pericardiotomy done drained approximately $550 \mathrm{mls}$ of blood. A size $36 \mathrm{Fr}$ pericardial drain was inserted and sternotomy closed with a size $7 \mathrm{G}$ stainless wire and skin closed in layers after haemostasis. Time of presentation to operating theatre was approximately 20 hours.

Patient made an uneventful recovery with the removal of the pericardial drain on postoperative day 4 . He was discharged home on postoperative day 7 for fol- 
low-up on out-patient basis. He was reviewed in two weeks and after two months after discharge on outpatient basis and he has been well without any complications.

\section{Case 2}

A 56-year-old male was rushed in to the accident and emergency centre of the Komfo AnokyeTeaching Hospital in Ghana by neighbours with the story that the patient had allegedly been shot by thieves. He was found semiconscious by the roadside and immediately rushed to the hospital. On arrival, he was pale and semi-conscious with GCS 10/15, pupils equal bilaterally but unreactive to light. Blood pressure on arrival was unrecordable but he had palpable femoral pulse, pulse rate of $100 \mathrm{bpm}$ which was weak and thready with distended neck veins and distant heart sounds. There were multiple pellets penetrating the chest at the precordium. Respiratory rate was 34 cycles per minute with unrecordable $\mathrm{SpO}_{2}$ and there was stony dullness on the left chest with markedly diminished air entry. The abdomen was full, soft and non-tender with no evidence of haemoperitoneum.

Patient was immediately started on fluid resuscitation but suddenly went into cardiac arrest for which CPR was started. Return of spontaneous cardiac activity was achieved after 15 mins. He was then sedated, intubated and a size 32FR left chest tube inserted. Vitals rechecked after 1.5 L I.V. fluids and 2 units of whole blood transfusion were BP 70/40 mmHg, PR 112 bpm (regular, moderate volume), urine output $40 \mathrm{mls} / \mathrm{hr}$ and $\mathrm{SpO}_{2} 98 \%$ - $100 \%$ on volume control mechanical ventilation. He was then immediately prepared for exploratory median sternotomy. Laboratory investigations done showed haemoglobin level of 11.7 $\mathrm{g} / \mathrm{dl}$, platelet count of $116 \times 10^{3} / \mathrm{uL}$ and white cell count of $10.10 \times 10^{3} / \mathrm{uL}$. Renal function test was normal.

Consent was then taken from relatives for exploratory median sternotomy. Under general anaesthesia with cuffed endotracheal intubation and patient in supine position, the chest was entered via a median sternotomy. The findings included:

- Left haemothorax of 2 litres

- Moderate haemopericardium of approximately $300 \mathrm{mls}$

- A $0.5 \mathrm{~cm}$ laceration of the pericardium at the diaphragmatic border

- A $1 \mathrm{~cm}$ laceration at the lateral border of the right upper lung lobe

- A pinhole right ventricular perforation with haematoma

- Mediastinal haematoma

Pericardiotomy was done and the ventricular perforation repaired with prolene 5/0. Haemothorax was evacuated and lung laceration repaired with vicryl 4/0. Mediastinal haematoma evacuated and haemostasis secured. A size 36Fr mediastinal tube was placed in situ and the sternotomy incision closed in layers with a size 6FG sternal wire. The initially passed thoracostomy tube was repositioned. Immediate post-operative period was uneventful. 
Early postoperative period was unremarkable until day 5 post-op when he started complaining of blurry vision for which an ophthalmologist consult was sought. On review, BP checked was 200/130 mmHg. He was also found to have features suggestive of bilateral ischemic optic damage.

The patient's blood pressure was gradually controlled on anti-hypertensives and vision returned to normalcy after 4 days. A head CT scan done showed no evidence of acute brain parenchymal disease. He was eventually discharged home on postoperative day 12 and followed up on out-patient basis. The time of presentation to surgery was 4 hours and 23 minutes and he had a total of 5 units of blood transfusion. He was reviewed on the first week after discharge and then in one month, 2 months and 6 months and so far he has no reported complications and he has been carrying out his normal duties (Figures 1-2).
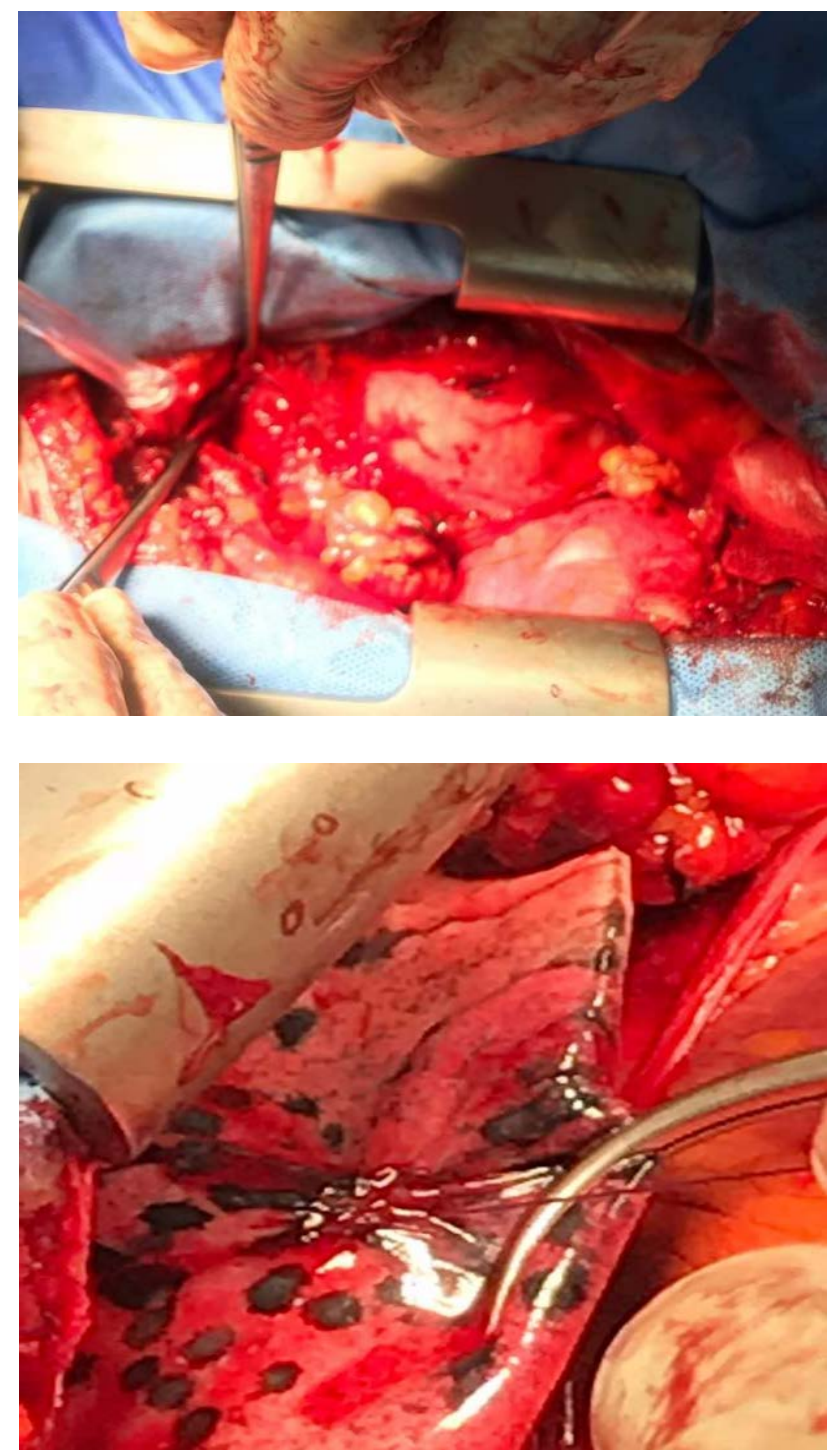

Figure 1. Intraoperative picture showing the inferior or diaphragmatic pericardial laceration on the left and the repaired right upper lobe lung. 

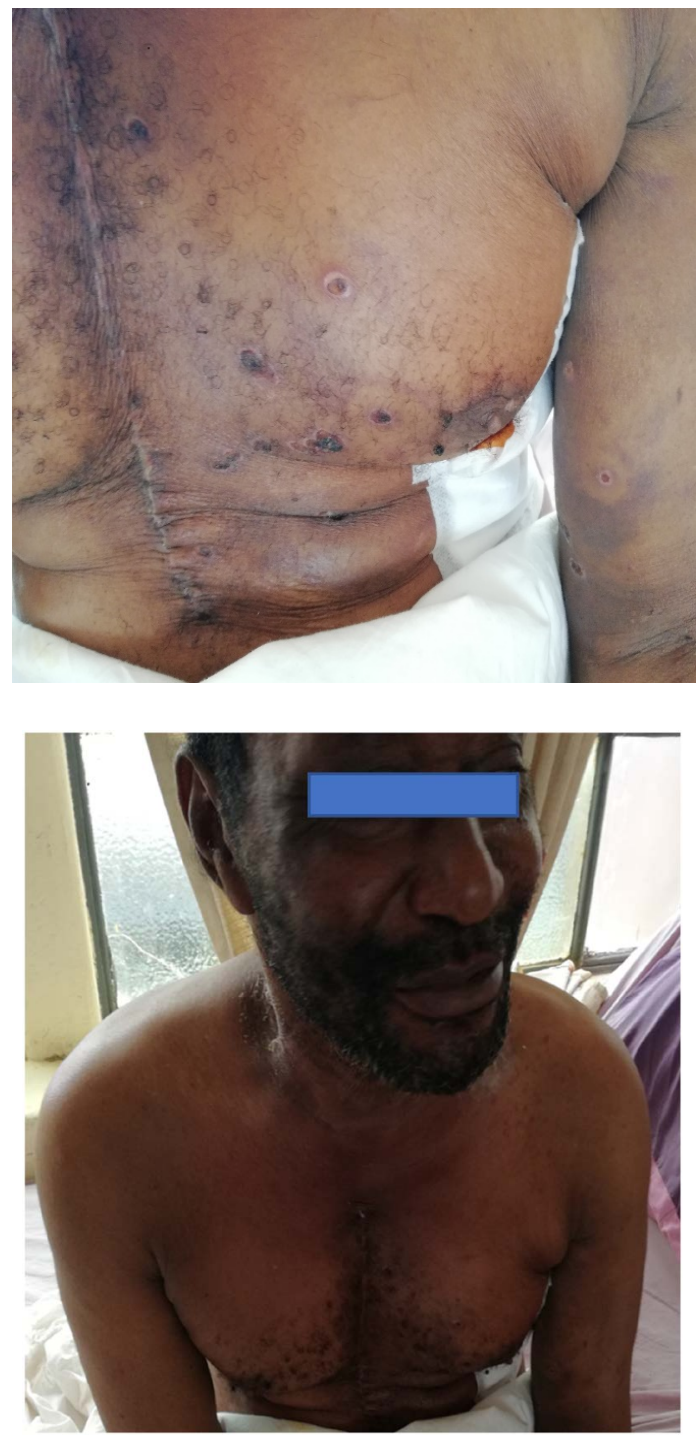

Figure 2. Postoperative period showing the sternotomy scar and the gunshot pellets wounds.

\section{Discussion}

Cardiac tamponade is a life-threatening hemodynamically significant compression of the heart by a sudden or gradual accumulation of collections in the pericardial space that incites and overrides the body's compensatory mechanism. Collections may range from air, solids or fluids such as blood, clots, pus or serous fluid [3] [4]. It is a major cause of pre-hospital deaths following chest trauma. According to Araujo et al. in 2016 in Manaus, 86.2\% out of 138 cases reported with cardiac trauma died at the scene. It is mainly as a result of cardiac chamber rupture following traumatic cardiac injury with the right-sided chambers been the most commonly implicated in literature [2] [5] as found in this case where there was a pinhole laceration of the right ventricle. This was in contrast to the observation by Araujo et al., who reported that the left ventricle was the most commonly affected side of cardiac trauma while considering single lesions. Ac- 
cording to the National Trauma Data Bank of the United States of America, cardiac chamber ruptures in traumatic cardiac injuries was associated with a high mortality rate of almost 90\% [2]. A rare case of cardiac tamponade was reported by Ryu and Lee resulting from an aortic injury [5]. Also reported in literature is a case of cardiac tamponade having occurred due to perforation of the pulmonary artery [6].

The pathophysiology of cardiac tamponade is that of a sudden or slowly progressing compression of the cardiac chambers as a result of an increase in the intra-pericardial pressure by accumulation of pericardial contents. In the event of a traumatic cardiac injury, the accumulated blood initially fills up the available reserve volume. The rate of blood collection in the pericardial cavity relative to the pericardium's ability to stretch determines the acute effect on the heart. In sudden pericardial space accumulations, the heart is constricted due to the fixed pericardial volume resulting in reduced venous return to the right heart as a consequence of severely diminished cardiac chamber volume. Consequently, there is a significant reduction in stroke volume due to the diminished pulmonary venous return. In the event of a cardiac chamber rupture or intra-pericardial great vessel injury with associated sudden leakage of blood into the pericardial cavity, the relatively stiff pericardium would result in severe life-threatening hemodynamic instability even with relatively very little blood collected [3] [4] [6]. About $200 \mathrm{mls}$ of pericardial fluid is required to accumulate in a normal pericardium to cause a tamponade. In chronic cases however, larger volumes are required [4]. Our first patient had $550 \mathrm{mls}$ of blood in the pericardial cavity whereas the second patient had approximately $330 \mathrm{mls}$ probably because of the presence of the pericardial laceration leading to blood leaking into the mediastinum and the left pleural space resulting in the 2 litres of the blood evacuated from the left pleural space.

Clinical detection of pericardial tamponade is particularly difficult as associated symptoms are usually non-specific especially in polytrauma patients [3] [5] [7]. However, chest pain is the most commonly reported symptom as reported by Telich-Tarriba and compatriots [2] and chest pains was the major complaint of our two patients. Physical findings alone are inadequate to accurately diagnose cardiac tamponade [7]. However, the location of chest wounds may provide a clue as to the likelihood of a cardiac injury as happened in our two cases where the gunshot wounds were mostly on the precordium and therefore injuring the heart, pericardium and the left pleural space but the second patient also sustained a right lung injury. The chest wall may be divided into the central and peripheral zones. The central zone-bordered superiorly by the medial halves of the clavicles and jugular notch, laterally by the imaginary midclavicular lines and inferiorly by the xiphoid process and costal cartilages-is deemed the danger zone as injuries to this area is likely to involve the heart [6]. In a case series by Crawford et al., all cases with injuries sustained in the danger zone had cardiac involvement with pericardial tamponade [6]. The Beck's triad-hypotension, muffled heart sounds and distended neck veins-though a very useful clinical finding in diagnosing cardiac tamponade, has been found to have low sensitivity and 
specificity especially in polytrauma patients [5]. Physical examination may also show pulsus paradoxus, which is a drop in systolic blood pressure of more than $10 \mathrm{mmHg}$ on inspiration [3].

The use in the emergency department of bedside ultrasonography/EFAST is a rapid and efficient modality in diagnosing cardiac tamponade, despite its possible false negative results. They could be very useful in the hands of a skilled physician to obtain high sensitivity and specificity results [7] [8] [9]. A chest x-ray may show cardiomegaly only with accumulation of more than 200 mls of fluid. Electrocardiogram done in cardiac tamponade may show electrical alternans which is described as the variation in the amplitudes of each QRS complex. P waves may be found to be involved in this alternation and reversal of polarity in which case the sign is particularly for pericardial tamponade [3]. Echocardiography will demonstrate fluid in the pericardial cavity associated with right atrial compression or right ventricular collapse in diastole along with deviation of the interventricular septum to the left in inspiration [4]. Our two patients benefited from the use of EFAST to make the diagnosis at the emergency department.

The options for management of acute traumatic cardiac tamponade are pericardiocentesis, either needle or catheter pericardiocentesis, subxiphoid tube pericardiostomy, subxiphoid pericardial window, pericardial window via left thoracotomy or median sternotomy [7]. For haemodynamically stable patients, haemopericardium may be managed conservatively with pericardiocentesis or sub-xiphoid pericardial window so long as haemopericardium remains non-clotted [8]. Pericardiocentesis is also useful in unstable patient being prepared for surgery who suddenly go into cardiac arrest. It may be employed to relieve some of the effect of the tamponade as part of the resuscitation [9]. The two cases were all rushed to theatre without pericardiocentesis probably because early diagnosis was made from the eFAST and the fact that they had become unstable and therefore had to be rushed for surgery after quick resuscitation with I.V fluids, as the second patient had to be intubated at the emergency department before being wheeled to theatre. Puncture sites utilized in pericardiocentesis include the subxiphoid, apical and parasternal approaches [7]. In the event of a pericardial clot, a subxiphoid pericardiotomy may not even be beneficial as reported by Ishida and associates in a case report [8].

For patients with suspected cardiac rupture, exploration via median sternotomy or left anterolateral thoracotomy is required [8]. Median sternotomy is the documented gold standard approach for evaluation of patients with cardiac injuries because it offers extensive access to the heart, great vessels and the pleural cavities. However, a left anterolateral thoracotomy incision is a faster and simpler approach easily utilized in emergency department thoracotomy for unstable patients who go into arrest and would benefit from an open chest cardiac massage since it provides easy access to the ventricles and pulmonary arteries [10]. We used median sternotomy for both patients because of the gunshots on the precordium with the suspicion of cardiac injuries which were confirmed intraoperatively with the second patient having a right ventricular perforation. 


\section{Conclusion}

Lifesaving emergency median sternotomy in chest injuries resulting in cardiac tamponade from acute haemopericardium is possible even in less-resourced hospitals especially if early diagnosis can be made and the services of cardiothoracic surgery are available.

\section{Acknowledgements}

Written informed consent was obtained from the patients for publication of these case reports and accompanying images and we are grateful to the theatre staff at the Accident and Emergency Centre.

\section{Conflict of Interest}

The authors declare no conflicts of interest regarding the publication of this paper.

\section{References}

[1] de Araújo, A.O., Westphal F.L., de Lima, L.C., de Oliveira Correia, J., Gomes P.H., Costa, E.N., et al. (2018) Fatal Cardiac Trauma in the City of Manaus, Amazonas State, Brazil. Revista do Colégio Brasileiro de Cirurgióes, 45, 1-8. https://doi.org/10.1590/0100-6991e-20181888

[2] Telich-Tarriba, J.E., Anaya-Ayala, J.E. and Reardon, M.J. (2012) Surgical Repair of Right Atrial Wall Rupture after Blunt Chest Trauma. Texas Heart Institute Journal, 39, 579-581.

[3] Spodick, D.H. (1960) Acute Cardiac Tamponade. Minerva Anestesiologica, 26, 166-171.

[4] Sellke, F.W., del Nido, P.J. and Swanson, S.J. (2016) Sabiston \& Spencer Surgery of the Chest. 9th Edition, Elsevier Inc., Philadelphia, 113-115.

[5] Ryu, D.W. and Lee, M.K. (2017) Cardiac Tamponade Associated with Delayed Ascending Aortic Perforation after Blunt Chest Trauma: A Case Report. BMC Surgery, 17, Article number: 70. https://doi.org/10.1186/s12893-017-0266-2

[6] Crawford, R., Kasem, H. and Bleetmen, A. (1997) Traumatic Pericardial Tamponade: Relearning Old Lessons. Emergency Medicine Journal, 14, 252-254. https://doi.org/10.1136/emj.14.4.252

[7] Otsuka, H., Sato, T., Morita, S., Nakagawa, Y. and Inokuchi, S. (2016) A Case of Blunt Traumatic Cardiac Tamponade Successfully Treated by Out-of-Hospital Pericardial Drainage in a "Doctor-Helicopter" Ambulance Staffed by Skilled Emergency Physicians. The Tokai Journal of Experimental and Clinical Medicine, 41, 1-3.

[8] Ishida, K., Kinoshita, Y., Iwasa, N., Nakae, M., Sakaki, M., Ieki, Y., et al. (2017) Emergency Room Thoracotomy for Acute Traumatic Cardiac Tamponade Caused by a Blunt Cardiac Injury: A Case Report. International Journal of Surgery Case Reports, 35, 21-24. https://doi.org/10.1016/j.ijscr.2017.03.009

[9] MacFarlane, C. (2005) Blunt Trauma Cardiac Tamponade: What Really Counts in Management. Emergency Medicine Australasia, 17, 416-417. https://doi.org/10.1111/j.1742-6723.2005.00792.x

[10] Cottini, M., Pergolini, A., Ranocchi, F. and Musumeci, F. (2018) The Role of Heart Team Approach in Penetrating Cardiac Trauma: Case Report and Review of the Literature. Brazilian Journal of Cardiovascular Surgery, 33, 99-103. https://doi.org/10.21470/1678-9741-2017-0150 